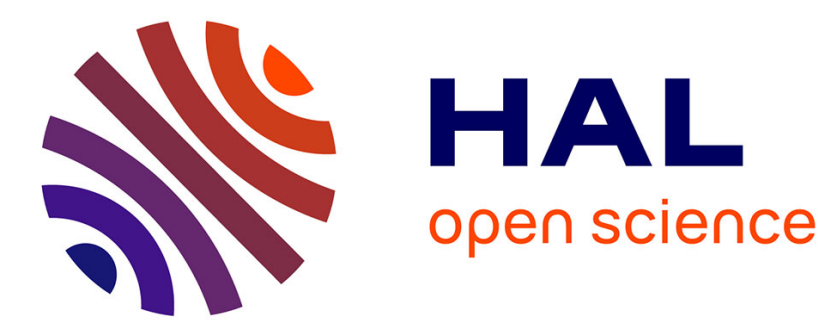

\title{
Irreversible Dynamics of Vortex Reconnections in Quantum Fluids
}

\author{
Alberto Villois, Davide Proment, Giorgio Krstulovic
}

\section{To cite this version:}

Alberto Villois, Davide Proment, Giorgio Krstulovic. Irreversible Dynamics of Vortex Reconnections in Quantum Fluids. Physical Review Letters, 2020, 125, 10.1103/physrevlett.125.164501 . hal03054354

\section{HAL Id: hal-03054354 \\ https://hal.science/hal-03054354}

Submitted on 11 Dec 2020

HAL is a multi-disciplinary open access archive for the deposit and dissemination of scientific research documents, whether they are published or not. The documents may come from teaching and research institutions in France or abroad, or from public or private research centers.
L'archive ouverte pluridisciplinaire HAL, est destinée au dépôt et à la diffusion de documents scientifiques de niveau recherche, publiés ou non, émanant des établissements d'enseignement et de recherche français ou étrangers, des laboratoires publics ou privés. 


\title{
Irreversible Dynamics of Vortex Reconnections in Quantum Fluids
}

\author{
Alberto Villois (1) \\ Department of Physics, University of Bath, Bath BA2 7AY, United Kingdom \\ and School of Mathematics, University of East Anglia, Norwich Research Park, Norwich NR4 7TJ, United Kingdom \\ Davide Proment(i) \\ School of Mathematics, University of East Anglia, Norwich Research Park, Norwich NR4 7TJ, United Kingdom \\ Giorgio Krstulovic (1) \\ Université Côte d'Azur, Observatoire de la Côte d'Azur, CNRS, Laboratoire Lagrange, \\ Bd de l'Observatoire, CS 34229, 06304 Nice cedex 4, France
}

(Received 7 May 2020; revised 30 June 2020; accepted 10 September 2020; published 15 October 2020)

\begin{abstract}
We statistically study vortex reconnections in quantum fluids by evolving different realizations of vortex Hopf links using the Gross-Pitaevskii model. Despite the time reversibility of the model, we report clear evidence that the dynamics of the reconnection process is time irreversible, as reconnecting vortices tend to separate faster than they approach. Thanks to a matching theory devised concurrently by Proment and Krstulovic [Phys. Rev. Fluids 5, 104701 (2020)], we quantitatively relate the origin of this asymmetry to the generation of a sound pulse after the reconnection event. Our results have the prospect of being tested in several quantum fluid experiments and, theoretically, may shed new light on the energy transfer mechanisms in both classical and quantum turbulent fluids.
\end{abstract}

DOI: 10.1103/PhysRevLett.125.164501

Introduction.-Irreversibility emerges naturally in most interacting systems characterized by a huge number of degrees of freedom. Its manifestation is associated to a time-symmetry breaking: the arrow of time appears inherently defined in the dynamics and an experienced observer is able to distinguish what are before and after.

In dissipative systems the arrow of time naturally reflects the dynamics that minimizes the energy. Classical viscous fluids present valuable examples. When no external forces are applied, an initial laminar flow decays in time until its kinetic energy is totally converted into heat. A less simple example is the particle pair dispersion in turbulent flows. Although two tracers separate from each other backward and forward in time with the same Richardson scaling, their rates are different [1]: particles separate slower forward in time than backward.

Conservative (energy-preserving) systems are more subtle. The arrow of time is defined only in a statistical sense by exploiting an entropy function that approaches its extremal as time progresses. The simplest example of this kind is the free-expansion experiment of a gas: even if the gas particles interact microscopically through conservative collisions, on average their macroscopic position and velocity distribution obeys the Boltzmann kinetic equation which is time irreversible.

Quantum fluids are exotic types of fluids characterized by the total absence of viscosity, thus being conservative. Examples of such systems are superfluid liquid helium [2] and Bose-Einstein condensates (BECs) made of dilute gases of bosons [3], Cooper-paired fermions [4], or massive photons [5]. As a consequence of the wave nature of their bosonic constituents, quantum fluids have two striking properties: vortices arise as topological defects in the order parameter and their circulation takes only discrete multiples of the quantum of circulation $\Gamma=h / m$, where $h$ is the Planck constant and $m$ is the boson's mass. These defects, referred to in the following as vortex filaments, present a complicated dynamics which still misses a general solution. A key point in such dynamics is the occurrence of reconnection events. A vortex reconnection is the process of interchange of two sections of different filaments; see the sketch in Fig. 1(a). It happens at small spatial and fast timescales [6], and allows the filament topology to vary.

For the sake of simplicity, we consider in this Letter a quantum fluid described by a single scalar order parameter. In the limit of zero temperature, this quantum fluid accommodates only two distinct excitation families: vortex-type excitations, in the form of filaments, and compressible density-phase excitations, that is sound waves. While the full dynamics is energy preserving, the energy may continually flow between these two excitation families. In this perspective, we provide a statistical analysis over many realizations of vortex reconnections, unveiling an inherent irreversible dynamics of the reconnection process. Moreover, we show how the linear momentum and energy transfers, from vortex-type 
(a)

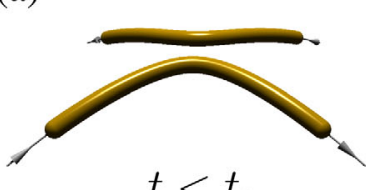

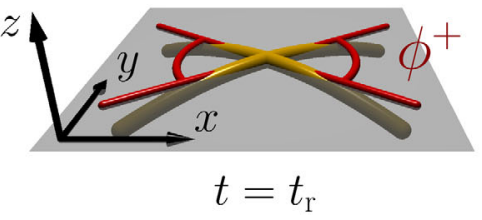
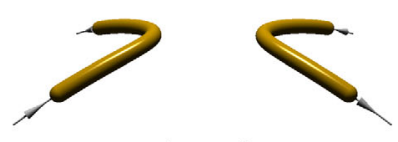

(b)

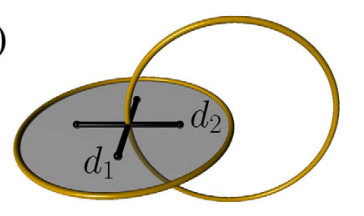

FIG. 1. (a) Sketch of a vortex reconnection event in quantum fluids. At the reconnection time $t_{r}$ the reconnecting filaments are locally tangent to the plane $x O y$, here depicted in gray, and form the reconnecting angle $\phi^{+}$. The vorticity of the filaments is depicted with gray arrows. (b) The Hopf link initial condition used to create the different realizations, with visual indication of the offset parameters $\left(d_{1}, d_{2}\right)$.

excitations to compressible density-phase excitations, are related to the geometrical parameters (macroscopic reconnection angle and concavity parameter) of the reconnecting filaments, explaining the origin of such irreversibility.

Main results.-We choose an initial configuration characterized by a Hopf link vortex filament, see Fig. 1(b), where (almost) all the superfluid kinetic energy is stored into the vortex-type excitations. Similarly to vortex knots, the Hopf links naturally decay into topologically simpler configurations [7-9] by performing a set of vortex reconnections. To study the Hopf link evolution, we use the Gross-Pitaevskii (GP) model, a nonlinear partial differential equation formally derived to mimic the order parameter $\psi$ of a BEC made of dilute locally interacting bosons, but qualitatively able to mimic a generic quantum fluid [10]. The GP equation, cast in terms of the healing length $\xi$ and the sound velocity $c$, reads

$$
i \frac{\partial \psi}{\partial t}=\frac{c}{\sqrt{2} \xi}\left(-\xi^{2} \nabla^{2} \psi+\frac{m}{\rho_{0}}|\psi|^{2} \psi\right),
$$

where $\rho_{0}$ is the bulk superfluid density and $m$ the mass of a boson. When the GP equation is linearized about the uniform bulk value $\left|\psi_{0}\right|=\sqrt{\rho_{0} / m}$, dispersive effects arise at scales smaller than $\xi$ and (large-scale) sound waves effectively propagate at speed $c$. In this Letter lengths and times are expressed in units of $\xi$ and $\tau=\xi / c$, respectively. Thanks to the Madelung transformation, $\psi(\mathbf{x}, t)=$ $\sqrt{\rho(\mathbf{x}, t) / m} \exp [i \phi(\mathbf{x}, t) /(\sqrt{2} c \xi)]$, Eq. (1) can be interpreted as a model for an irrotational inviscid barotropic fluid of density $\rho$ and velocity $\mathbf{v}=\nabla \phi$. Vortices arise as topological defects of circulation $\Gamma=h / m=2 \sqrt{2} \pi c \xi$ and vanishing density core size order of $\xi$ [11]. In the previous formula, $h$ is the Planck constant.

We evolve a Hopf link prepared by superimposing two rings of radius $R=18 \xi$, obtained by using a NewtonRaphson and biconjugate-gradient technique [12] to ensure a minimal amount of compressible energy; details on the numerical scheme and on the generation of the initial condition are in the Supplemental Material [13]. A set of 49 different realizations are obtained by changing the offsets $\left(d_{1}, d_{2}\right)$ of one ring as sketched in Fig. 1(b), taking $d_{i} \in$ $[-9 \xi, 9 \xi]$ with unit step of $3 \xi$. During the evolution one or more reconnection events occur. It has been shown [14-17] that about the reconnection event, the distance between the two filaments behaves as

$$
\delta^{ \pm}(t)=A^{ \pm}\left(\Gamma\left|t-t_{r}\right|\right)^{1 / 2}
$$

where $A^{ \pm}$are dimensionless prefactors and $t_{r}$ is the reconnection time; the superscripts - and + label the cases before and after the reconnection, respectively. In each Hopf link realization, we carefully track [18] all reconnecting events and measure $A^{ \pm}$. The measured values of $\delta^{2}(t)$ for all the 71 analyzed reconnections are shown in Fig. 2; the best-fit $A^{ \pm}$are plotted in red dots in the inset of Fig. 2 . Remarkably, the reconnecting filaments always separate faster (or at an almost equal rate) than they approach; that is, $A^{+} \geq A^{-}$. The clear asymmetry recorded in the $\delta^{2}$ versus $t-t_{r}$ and in the distribution of the $A^{ \pm}$'s is the fingerprint of the irreversible dynamics characterizing the vortex reconnection process. For completeness, we also report in the inset of Fig. 2, using different symbols, the prefactor measurements obtained in previous works $[15,16]$, which corroborate even further our results. Finally note that in a

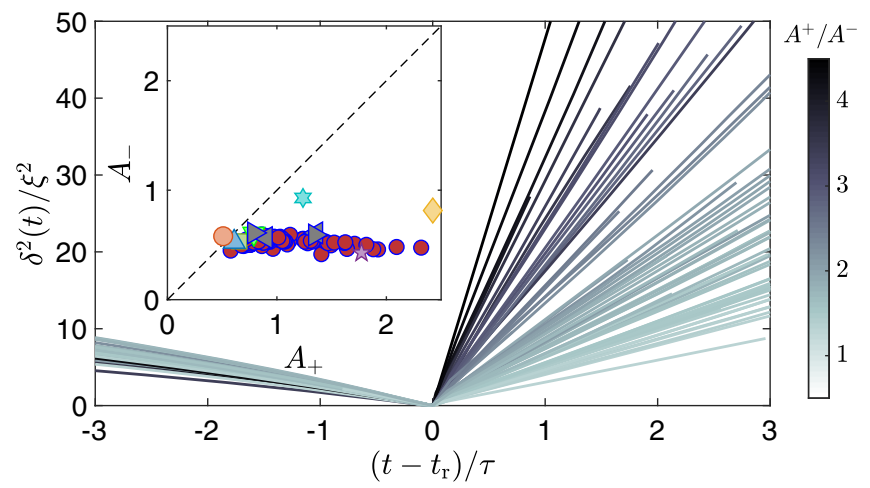

FIG. 2. Squared distances versus time of the reconnecting filaments measured in all the 49 realizations. The gray-scale color indicates the estimated value of $A^{+} / A^{-}$in each case. Inset: values of approach and separation prefactors $A^{+}$and $A^{-}$. Red points correspond to data of the present work. Gray left- and right-facing triangles correspond to reconnections of free and trapped vortices, respectively, from Galantucci et al. [16]; other symbols from Villois et al. [15]. 
recent work [19], it has been reported that vortex reconnections in the Navier-Stokes equation also display a clear $t^{1 / 2}$ scaling, a coefficient $A^{-} \sim 0.3-0.4$ and the same time asymmetry $A^{+}>A^{-}$. Note that the Biot-Savart (BS) analytical calculations of Ref. [20] and the local induction approximation based ones of Ref. [21] predict $A^{-} \sim 0.47$ and $A^{-}=0.427$, respectively, which are in agreement with our GP measurements. In what follows, we quantitatively relate the asymmetry in the distribution of the prefactors with the irreversible energy transfer between the vortextype and density or phase excitation families occurring during a reconnection event. Previous numerical studies of the GP model have indeed reported the clear emission of a sound pulse during reconnection events [22,23]. A series of snapshots showing the sound pulse emitted during the decay of the Hopf link in one of our realizations is reported in Ref. [24].

The simple linear theory neglecting the nonlinear term of the GP model $[14,15]$, valid in the limit $\delta^{ \pm} \rightarrow 0$, provides insight into the dynamics of reconnecting parameters as the order parameter can be found analytically. It predicts that the filaments reconnect tangent to a plane, in our reference frame the $z=0$, see Fig. 1(a), and that the projections of the filaments onto it approach and separate following the branches of a hyperbola. The macroscopic (post) reconnection angle, formed by the hyperbola asymptotes, results in

$$
\phi^{+}=2 \operatorname{arccot}\left(A_{r}\right), \quad \text { where } A_{r}=A^{+} / A^{-} .
$$

the projections of the filaments onto the orthogonal plane $y=0$ form a parabola (not shown in here; see Ref. [24] for more details). Without any loss of generality, we set $\Lambda / \zeta$ the concavity of such a parabola, and we refer to $\Lambda$ as the concavity parameter, where $\zeta$ is an arbitrary length scale, whose value is not important in the following discussion.

In all the reconnection events detected, we observe a distinct sound pulse generated after the reconnection and propagating toward the positive $z$ axis, as shown in Figs. 3(a) and 3(b). Figure 3(c) shows the behavior of the superfluid density along the $z$ direction versus times $t-t_{r}$. A (depression) sound pulse is generated soon after the reconnection and propagates toward the positive $z$ direction at a speed qualitatively close to the speed of sound in the bulk; refer to the green dashed line $z=c\left(t-t_{r}\right)$, with $c$ defined in Eq. (1). Note that the other low density regions, corresponding to the density depletions of the vortex cores, move much slower.

To explain the generation and directionality of such a pulse, we devise a novel theoretical approach, detailed in Ref. [24], and summarize in the following. Let us denote by $\mathbf{R}_{1}^{ \pm}(s, t)$ and $\mathbf{R}_{2}^{ \pm}(s, t)$ the reconnecting filaments, with $s$ being their spatial parametrization variable. Far from the reconnection point (both before and after), the dynamics of the vortex filaments are mostly driven by
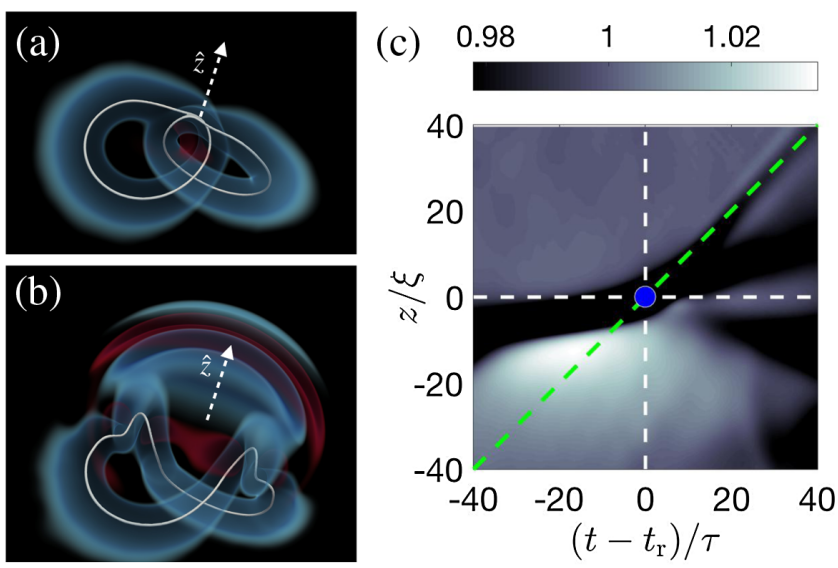

FIG. 3. Three-dimensional rendering of the density field. White contours display the vortices and density fluctuations are rendered in blue-redish colors: (a) reconnection time and (b) at $t-t_{r} \approx 40 \tau$. (b) The positive direction of the $z$ axis is also depicted with a white arrow. (c) Spatiotemporal plot of density along the $z$ axis about the reconnection event denoted by the blue central point. The two dashed green lines are $z=c\left(t-t_{r}\right)$; here, the reconnection point $(0,0)$ is represented by the blue dot.

the Biot-Savart model, which describes the motion of $\delta$-supported vorticity in an incompressible inviscid flow [25]; note that this limit can be formally derived from GP [26]. In our realizations, BS is valid at distances $\delta^{ \pm}(t) \gg \delta_{\text {lin }}$, whereas for $\delta^{ \pm}(t) \ll \delta_{\text {lin }}$ the dynamics is determined by the linear approximation, given $\delta_{\text {lin }}$ is a crossover scale of order of the healing length. We assume both descriptions approximately valid when the filaments are at the distance $\delta^{ \pm}\left(t^{ \pm}\right) \approx \delta_{\text {lin }}$. This hypothesis, validated by previous GP simulations [15,16], allows us to perform an asymptotic matching.

We can therefore compute the difference, before and after the reconnection, of BS linear momentum $\Delta \mathbf{P}_{\text {fil }}$ using the positions of the filaments $\mathbf{R}_{1}^{ \pm}\left(s, t^{ \pm}\right)$and $\mathbf{R}_{2}^{ \pm}\left(s, t^{ \pm}\right)$ coming from the linear approximation. As shown in Ref. [24], note that these depend only on the reconnection angle $\phi^{+}$(or equivalently $A_{r}$ ) and the concavity parameter $\Lambda$. Within BS, the linear momentum is given as the line integral $\mathbf{P}_{\text {fil }}(t)=\left(\rho_{0} / 2\right) \Gamma \oint \mathbf{R}(s, t) \times d \mathbf{R}(s, t)$ [27]. As the total linear momentum of the superfluid is conserved in GP [28], the linear momentum carried by the sound pulse created after the reconnection must compensate the loss of linear momentum accounted by $\Delta \mathbf{P}_{\text {fil }}$ and reads [24]

$$
\mathbf{P}_{\text {pulse }}=-\Delta \mathbf{P}_{\text {fil }} \propto\left(0,0,2 \csc \phi^{+}\right)
$$

independently of the $\delta_{\operatorname{lin}}$ chosen. This result is remarkable: the sound pulse linear momentum is (overall) nonzero only in the positive $z$ direction, as observed in all our reconnection events, and its amplitude is independent of $\Lambda$ and minimal for $\phi^{+}=\pi / 2$. 


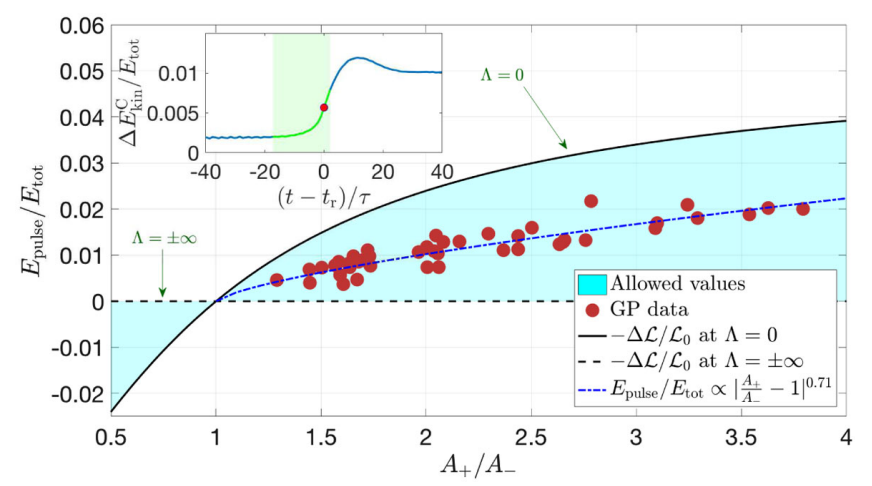

FIG. 4. Relative energy transferred to waves during the reconnection process. The cyan zone denotes the allowed values from the matching theory. Inset: relative increase of compressible kinetic energy (solid blue) about a reconnection event (denoted by the red dots) for a typical realization. The green area corresponds to the interval defined by $\delta^{ \pm}(t) \leq \delta_{\operatorname{lin}}=6 \xi$.

The same matching theory can be applied to estimate the amount of energy transferred to the sound pulse. Following the standard energy splitting protocol in GP [29], the superfluid kinetic energy is decomposed into a compressible component $E_{\text {kin }}^{C}$, associated to sound excitations, and an incompressible component $E_{\text {kin }}^{I}$, associated to vortextype excitations. In all our realizations, we observe a sharp growth of $E_{\text {kin }}^{C}$ during each reconnection event. An example of its evolution, normalized by the total (constant) energy $E_{\text {tot }}$, is shown in the inset of Fig. 4: here the red dot indicates the reconnection time and the green region indicates the times when $\delta^{ \pm}(t) \leq \delta_{\text {lin }}=6 \xi$. The increase of $E_{\text {kin }}^{C}$ during the reconnection event is related to the loss of incompressible kinetic energy $E_{\text {kin }}^{I}$. For all the reconnection events measured in our realizations, we compute the energy transferred to the sound pulse as $E_{\text {pulse }}=-\Delta E_{\text {kin }}^{I}$, where $\Delta E_{\text {kin }}^{I}=E_{\text {kin }}^{I}\left(t^{+}\right)-E_{\text {kin }}^{I}\left(t^{-}\right)$. Figure 4 shows the measured $E_{\mathrm{pulse}} / E_{\mathrm{tot}}$ data versus $A_{r}$ : there is clear correlation between these two quantities, with a best-fit scaling of $E_{\text {pulse }} / E_{\text {tot }} \propto$ $\left(A_{r}-1\right)^{0.71}$.

To the simplest approximation, called local induction approximation, the BS superfluid kinetic energy is proportional to the total length of the filaments. As the representations $\mathbf{R}_{1}(s, t)$ and $\mathbf{R}_{2}(s, t)$ have infinite lengths (as in the linear regime they do not close) we choose to account only for the length of finite sections of the filaments contained in a cylinder of radius $R \gg \delta_{\text {lin }}$, centered at the reconnection point and parallel to the $z$ axis. Evaluating $E_{\text {pulse }}$ reduces thus to the computation of the difference $\Delta \mathcal{L}\left(A_{r},|\Lambda| / \zeta, \delta_{\text {lin }}, R / \delta_{\text {lin }}\right)$ of the length of these sections; see [24] for more details. As the total GP energy is conserved, we have that

$$
E_{\text {pulse }} / E_{\text {tot }}=-\Delta \mathcal{L} / \mathcal{L}_{0},
$$

given $\mathcal{L}_{0}$ is the initial length of the Hopf link filament. For any given choices of $\delta_{\text {lin }}$ and $R$, all the admissible values of the theoretical estimation $\Delta \mathcal{L}$, rendered in cyan color in Fig. 4, are bounded between two lines obtained setting $\Lambda=0$ (dashed line) and $|\Lambda| \rightarrow \infty$ (solid line). The GP data are all distributed within these admissible values, thus confirming the accuracy of the matching theory.

Remarkably, the estimation of $E_{\text {pulse }}$ explains in a straightforward way the time asymmetry between the rates of approach and separation reported in Fig. 2 and its inset. Independently on the value of the concavity parameter $\Lambda$, the energy of the sound pulse is only non-negative when $A^{+} \geq A^{-}$, meaning that unless energy is externally provided to the reconnecting vortices, it is energetically impossible to have a reconnection event where $A^{+}<A^{-}$, or equivalently, where $\phi^{+}>\pi / 2$.

Closing remarks.-In this Letter we reported numerical evidence of the irreversible dynamics of vortex reconnections in a scalar quantum fluid and explain its origin thanks to a matching theory developed concurrently in Ref. [24]. This theory is based on very general physical considerations and give bounds for the energy of the pulse emitted during a reconnection event. However, it cannot determine the exact value of the reconnecting angle and, thus, the one of $A_{+} / A_{-}$. Our results can be extended to more complicated quantum fluids where nonlocal interactions and/or higher order nonlinearities are included, like BECs with dipolar interactions, cold Fermi gases, and superfluid liquid ${ }^{4} \mathrm{He}$.

In quantum fluid experiments, the detailed study of vortex reconnections is still in its infancy. In current BECs made of dilute gases, reconnecting vortices are created only in a nonreproducible way using fast temperature quenches [30]; however, new protocols have been proposed to create vortices in a reproducible manner [31]. In such setups, once the reconnection plane is identified, it should be feasible to measure the rates of approach and separation and detecting directionality of the sound pulse, using, for instance, Bragg spectroscopy [32]. In superfluid liquid ${ }^{4} \mathrm{He}$ experiments, vortex reconnections have been detected so far only at relatively high temperature where the normal component is non-negligible [33]. This latter may provide energy but also dissipates it through mutual friction; hence, measuring experimentally the distribution of the rates of approach and separation at different temperatures would be particularly desirable.

Finally, let us come back to the concept of irreversibility. In the realizations presented in this Letter, almost all of the superfluid kinetic energy is initially stored in the vortextype excitations. This is likely to cause the observed statistical asymmetry in the distribution of the rates of approach and separation to be maximized. At finite temperatures or in a turbulent tangle, fluctuations can provide extra energy to reduce this asymmetry, perhaps allowing also for $\phi^{+}>\pi / 2$, but the time asymmetry should in 
principle remain as an inherent mechanism allowing the system to reach the equilibrium. From a fluid dynamical point of view, let us remark that vortex reconnections are allowed and regular, in classical fluids due to the presence of viscosity, while in quantum fluids thanks to a dispersive term. Showing whether the resulting dynamics of these two different fluids are equivalent or not, in the limit where their respective regularization scale tends to zero, is an appealing open problem. Comparing the results presented in this Letter with a similar study in Navier-Stokes or a carefully regularized Biot-Savart model might provide some insight on the spontaneous stochasticity and the dissipative anomaly of turbulent flows, two concepts closely related to irreversibility.

The authors acknowledge L. Galantucci for providing some of the data displayed in the inset of Fig. 2. G. K., D. P., and A. V. were supported by the cost-share Royal Society International Exchanges Scheme (IE150527) in conjunction with CNRS. A. V. and D. P. were supported by the EPSRC First Grant scheme (EP/P023770/1). G. K. and D. P. acknowledge the Federation Doeblin for supporting D. P. during his sojourn in Nice. G. K. was also supported by the ANR JCJC GIANTE ANR-18-CE30-0020-01 and by the EU Horizon 2020 research and innovation programme under the Grant Agreement No. 823937 in the framework of Marie Sklodowska-Curie HALT project. Computations were carried out at Mésocentre SIGAMM hosted at the Observatoire de la Côte d'Azur and on the High Performance Computing Cluster supported by the Research and Specialist Computing Support service at the University of East Anglia. Part of this work has been presented at the workshop "Irreversibility and Turbulence" hosted by Fondation Les Treilles. G. K. and D. P. acknowledge Fondation Les Treilles and all participants of the workshop for the fruitful scientific discussions and support.

[1] J. P. Salazar and L. R. Collins, Annu. Rev. Fluid Mech. 41, 405 (2009).

[2] R. J. Donnelly, Quantized Vortices in Helium II (Cambridge University Press, Cambridge, England, 1991), Vol. 3.

[3] L. Pitaevskii and S. Stringari, Bose-Einstein Condensation and Superfluidity (Oxford University Press, New York, 2016), Vol. 164.

[4] A. J. Leggett, Quantum Liquids: Bose Condensation and Cooper Pairing in Condensed-Matter Systems (Oxford University Press, New York, 2006).

[5] I. Carusotto and C. Ciuti, Rev. Mod. Phys. 85, 299 (2013).

[6] Small length scales compared to the average filaments' length and fast timescales compared to the average filaments' length divided by their average speed.

[7] D. Proment, M. Onorato, and C. F. Barenghi, Phys. Rev. E 85, 036306 (2012).

[8] D. Proment, M. Onorato, and C. F. Barenghi, J. Phys. Conf. Ser. 544, 012022 (2014).
[9] D. Kleckner, L. H. Kauffman, and W. T. M. Irvine, Nat. Phys. 12, 650 (2016).

[10] A. Villois, D. Proment, and G. Krstulovic, Phys. Rev. E 93, 061103(R) (2016).

[11] L. Pitaevskii, Sov. Phys. JETP 13, 451 (1961), http://www .jetp.ac.ru/cgi-bin/e/index/e/13/2/p451?a=list.

[12] M. Abid, C. Huepe, S. Metens, C. Nore, C. T. Pham, L. S. Tuckerman, and M. E. Brachet, Fluid Dyn. Res. 33, 509 (2003).

[13] See Supplemental Material at http://link.aps.org/ supplemental/10.1103/PhysRevLett.125.164501 for specific details on the numerical scheme used, the numerical creation of the Hopf link, and the numerical analysis of each reconnection process.

[14] S. Nazarenko and R. West, J. Low Temp. Phys. 132, 1 (2003).

[15] A. Villois, D. Proment, and G. Krstulovic, Phys. Rev. Fluids 2, 044701 (2017).

[16] L. Galantucci, A. W. Baggaley, N. G. Parker, and C. F. Barenghi, Proc. Natl. Acad. Sci. U.S.A. 116, 12204 (2019).

[17] A. Enciso and D. Peralta-Salas, arXiv:1905.02467.

[18] A. Villois, G. Krstulovic, D. Proment, and H. Salman, J. Phys. A 49, 415502 (2016).

[19] J. Yao and F. Hussain, J. Fluid Mech. 900, R4 (2020).

[20] L. Boué, D. Khomenko, V. S. L'vov, and I. Procaccia, Phys. Rev. Lett. 111, 145302 (2013).

[21] S. Rica, C. R. Méc. 347, 365 (2019).

[22] M. Leadbeater, T. Winiecki, D. C. Samuels, C. F. Barenghi, and C. S. Adams, Phys. Rev. Lett. 86, 1410 (2001).

[23] S. Zuccher, M. Caliari, A. W. Baggaley, and C. F. Barenghi, Phys. Fluids 24, 125108 (2012).

[24] D. Proment and G. Krstulovic, companion paper, Phys. Rev. Fluids 5, 104701 (2020).

[25] K. W. Schwarz, Phys. Rev. B 38, 2398 (1988).

[26] M. D. Bustamante and S. Nazarenko, Phys. Rev. E 92, 053019 (2015).

[27] L. M. Pismen and L. M. Pismen, Vortices in Nonlinear Fields: From Liquid Crystals to Superfluids, From NonEquilibrium Patterns to Cosmic Strings (Oxford University Press, New York, 1999), Vol. 100.

[28] This is formally true in a system which is invariant under spatial translations. We can extend this property to a finite system if we assume that the boundaries are sufficiently far from the reconnection point so that the conservation of the linear momentum is almost exact within a given volume enclosing the reconnection event.

[29] C. Nore, M. Abid, and M. Brachet, Phys. Fluids 9, 2644 (1997).

[30] S. Serafini, M. Barbiero, M. Debortoli, S. Donadello, F. Larcher, F. Dalfovo, G. Lamporesi, and G. Ferrari, Phys. Rev. Lett. 115, 170402 (2015).

[31] K. Xhani, E. Neri, L. Galantucci, F. Scazza, A. Burchianti, K. L. Lee, C. F. Barenghi, A. Trombettoni, M. Inguscio, M. Zaccanti, G. Roati, and N. P. Proukakis, Phys. Rev. Lett. 124, 045301 (2020).

[32] J. Steinhauer, R. Ozeri, N. Katz, and N. Davidson, Phys. Rev. Lett. 88, 120407 (2002).

[33] G. P. Bewley, M. S. Paoletti, K. R. Sreenivasan, and D. P. Lathrop, Proc. Natl. Acad. Sci. U.S.A. 105, 13707 (2008). 